\title{
Recent Progress in Using Advanced Characterization and Modeling Approaches to Study Radiation Effects in Oxide Ceramics
}

\author{
XIAN-MING BAI ${ }^{1,2}$ \\ 1.-Idaho National Laboratory, 2525 N Fremont Avenue, P. O. Box 1625, MS 3835, Idaho Falls, \\ ID 83415-0001, USA. 2.-e-mail: xianming.bai@inl.gov
}

Oxide ceramics play a critical role in nuclear energy applications. For example, uranium dioxide $\left(\mathrm{UO}_{2}\right)$ and uranium-plutonium mixed oxide (MOX) are widely used as nuclear fuels in light water reactors (LWRs) to produce about $15 \%$ of the electricity in the world. Other oxide ceramics such as pyrochlores have been extensively studied as waste forms for the immobilization of nuclear waste. Similar to other materials, radiation induces complex microstructural evolution in oxide ceramics. In nuclear fuels, nuclear fission produces not only point defects and defect clusters but also many fission products such as xenon $(\mathrm{Xe})$ and krypton $(\mathrm{Kr})$. The evolution of these defects and fission products can form many extended defects such as dislocation loops, gas bubbles, and metallic precipitates. In turn, many physical properties such as thermal conductivity and mechanical strength degrade significantly. Pyrochlores are complex oxides and usually contain more than two types of cations (e.g., $\mathrm{A}$ and $\mathrm{B}$ cations in $\mathrm{A}_{2} \mathrm{~B}_{2} \mathrm{O}_{7}$ ). Under radiation, not only interstitials and vacancies are produced, but also cation antisite defects, in which some A and B cations exchange their positions. The accumulation of antisite defects can lead to amorphization and eventually degradation of the structural stability. Therefore, understanding the detailed information of radiation effects in these oxides is important for improving their performance for nuclear applications.

Radiation damage is a multi-timescale and multilengthscale problem. The timescale spans from picosecond to year, and the lengthscale spans from angstrom to meter. Therefore, understanding the radiation effects requires a multiscale approach,

Xian-Ming "David" Bai is the guest editor for the Nuclear Materials Committee of the TMS Structural Materials Division, and coordinated the topic Radiation Effects in Oxide Ceramics and Novel LWR Fuels in this issue. both experimentally and computationally. In particular, the information at the atomistic and mesoscale levels is crucial for us to understand the detailed defect and microstructural evolution under radiation. In this topic, five articles have been selected to show some recent progress in using advanced characterization and modeling approaches to understand the complex radiation effects in oxide ceramics with atomistic- and meso-scale resolution.

In the article by Desgranges et al., the authors used Raman spectroscopy to try to understand the defect structures in alpha particle irradiated $\mathrm{UO}_{2}$. Compared with pristine $\mathrm{UO}_{2}$, three new peaks, namely U1, U2, and U3, appeared in the Raman spectrum of the irradiated $\mathrm{UO}_{2}$. The appearance of new Raman peaks could be induced by resonant Raman, formation of new molecular entities, or breakdown in symmetry. The authors argued that the last one could be the predominant mechanism for inducing these new Raman peaks. To support this argument, the authors compared the Raman spectrum of irradiated $\mathrm{UO}_{2}$ with that of $\mathrm{U}_{4} \mathrm{O}_{9}$, which has a known crystal structure. The three peaks also appear in the spectrum of $\mathrm{U}_{4} \mathrm{O}_{9}$, although some peaks have much stronger intensities than those in the irradiated $\mathrm{UO}_{2} . \mathrm{U}_{4} \mathrm{O}_{9}$ contains some cuboctahedral oxygen interstitial clusters that have been identified in neutron diffraction experiments previously, ${ }^{1}$ and the charge state of some uranium ions is found to change from $4+$ to $5+{ }^{2}$ Accordingly, the coordination polyhedrons of uranium ions change from a cube for $\mathrm{U}^{4+}$ to a square antiprism for $\mathrm{U}^{5+}$, which can lead to high $\mathrm{U} 2$ and U3 peaks. The authors also discussed that doping of trivalent $(3+)$ cations can form octahedral polyhedrons, leading to the appearance of U1 and U2 peaks. Therefore, the authors proposed that radiation may change the charge state of $\mathrm{U}^{4+}$ to $\mathrm{U}^{5+}$ and $\mathrm{U}^{3+}$, and their corresponding coordination polyhedrons lead to the new peaks in Raman spectrum. 
Although the conclusion is not decisive, the work provides a good hypothesis that may be further investigated using a combined experimental and modeling approach in the future.

In the article by $\mathrm{He}$ et al., the authors used in situ transmission electron microscopy (TEM) to observe the defect evolution in the grain interior and near grain boundaries $(\mathrm{GBs})$ in polycrystalline $\mathrm{UO}_{2}$ irradiated by $\mathrm{Kr}$ ions at $600^{\circ} \mathrm{C}$ and $800^{\circ} \mathrm{C}$. The in situ observation allowed the authors to investigate the detailed process of nucleation and growth of dislocation loops in real time. Compared with single-crystal $\mathrm{UO}_{2},{ }^{3}$ the dislocation loop evolution in the grain interior of the polycrystal (note that the average grain size is about $5 \mu \mathrm{m}$ ) is similar: small loops $\rightarrow$ big loops $\rightarrow$ dislocation lines $\rightarrow$ dislocation tangles. However, the GBs in polycrystal were found to affect the local defect evolution significantly. For example, the authors observed a dislocation denuded zone near a GB at $800^{\circ} \mathrm{C}$. Although the observation of dislocation denuded zones in $\mathrm{Al}_{2} \mathrm{O}_{3}$ and $\mathrm{MgAl}_{2} \mathrm{O}_{4}{ }^{4}$ has been reported previously, to the best of my knowledge, such defect denuded zones have not been reported in $\mathrm{UO}_{2}$. Interestingly, the authors also found that the width of the denuded zone increased with increasing irradiation dose. However, no denuded zone was found near $\mathrm{GBs}$ at $600^{\circ} \mathrm{C}$ at the same irradiation dose, which suggests that the formation of a dislocation denuded zone is temperature sensitive. Although GBs in $\mathrm{UO}_{2}$ are expected to be defect sinks as in other materials, ${ }^{5}$ this study provides direct experimental evidence to support this expectation. This work demonstrates that GBs in $\mathrm{UO}_{2}$ can reduce the local defect concentration and suppress dislocation formation-an important property might be useful for future fuel design. However, there are still many questions to be answered such as the correlation between the denuded zone and GB character. Future systematic studies on this topic may elucidate these questions.

GBs are also preferential places for fission gas segregation. In the article by Valderrama et al., the authors used atom probe tomography (APT) to investigate the effects of GB structure on $\mathrm{Kr}$ segregation in $\mathrm{Kr}$-irradiated $\mathrm{UO}_{2}$. APT can provide atomic resolution of the species distribution in materials, so it is a powerful tool to study the fission gas segregation behavior at GBs. Using APT, the authors obtained the $\mathrm{Kr}$ concentration profiles across a high-angle GB $\left(46^{\circ}\right)$ and a low-angle GB $\left(14^{\circ}\right)$ at regions free of bubbles. At $1600^{\circ} \mathrm{C}$, the $\mathrm{Kr}$ concentration at the two GBs was a few times higher than in bulk, indicating $\mathrm{Kr}$ segregated to both GBs. Interestingly, the high-angle GB was found to contain about $35 \%$ more $\mathrm{Kr}$ than the lowangle GB, suggesting that the high-angle GB has a stronger segregation strength than the low-angle GB. This result provides the first direct experimental evidence to support the previous theoretical calculations of fission gas segregation at GBs in
$\mathrm{UO}_{2} \cdot{ }^{6}$ However, currently only two GBs have been studied in this work. In the future, with more statistics on different GBs, a clearer picture may emerge to understand the correlation between the fission gas segregation and GB character. Another interesting phenomenon observed by the authors is that grain growth is not significant in the $\mathrm{Kr}$-irradiated $\mathrm{UO}_{2}$ while it is evident in the nonirradiated $\mathrm{UO}_{2}$ upon high-temperature annealing. Their TEM observation showed that many intergranular bubbles formed at high temperatures in irradiated samples. As the authors argued, these bubbles may cause pinning effects on GB motion so that the grain growth was inhibited.

Radiation induces complex microstructures in oxide fuels. However, how they look like in three dimensions (3D) is rarely known. In addition, the microstructures developed in neutron-irradiated reactor fuels may be different from those in ionirradiated samples. However, the characterization of such highly radioactive fuels is prohibited in many laboratories because of safety regulation. In the article by Teague et al., the authors used the unique characterization facilities (electron backscatter diffraction, energy dispersive spectrometer, etc.) at Idaho National Laboratory (INL) that can handle radioactive samples to obtain $3 \mathrm{D}$ microstructures of high-burnup MOX fuels taken out from the Fast Flux Test Facility in the mid-1980s. Due to the thermal gradient in reactor fuels, the microstructures are quite different at the hot end and cold end. The authors found that the hot end contains larger grains, very large intergranular metallic precipitates, and some small pores. The pores have flat edges when they contact with precipitates. The cold end contains smaller grains, small intragranular metallic precipitates, and no pores. Even more interestingly, the authors reconstructed the experimentally determined 3D microstructures in INL's mesoscale MARMOT simulation package ${ }^{7}$ and used these microstructures to calculate the thermal conductivities of the two samples. Note that the MARMOT package is based on the phase field method coupled with many other physics such as heat conduction. Using MARMOT, they found that the $3 \mathrm{D}$ microstructures did lead to different thermal conductivities than the conventionally used 2D microstructures, although sometimes the difference is not significant depending on the details of microstructures. As expected, they also found that metallic precipitates increase the thermal conductivity while pores decrease the thermal conductivity. As mentioned by the authors, the resolution of the experimental characterization is about $200 \mathrm{~nm}$ so that some small microstructures below the resolution limit cannot be detected. As a result, such small defects were not considered in the modeling either. Therefore, the modeling work mainly evaluates the effects of different large microstructures on thermal conductivity. The phonon scattering effects caused by small defects, 
which may cause a significant impact on thermal conductivity, will be included in their future modeling study.

Radiation effects in pyrochlores are also very complex, ${ }^{8}$ partially due to the formation of cation antisite defects. In the article by Uberuaga et al., the authors used computational approaches to study the correlation between the radiation stability and the formation energies of antisite defects in $\mathrm{A}_{2} \mathrm{~B}_{2} \mathrm{O}_{7^{-}}$ type pyrochlores, where $\mathrm{B}$ represents the family of the pyrochlore. Radiation can mix A and B cations to form antisite defects (so-called disorder). If half of A cations are on B sites (and vice versa), complete disorder occurs. As disorder accumulates, amorphization eventually occurs. The authors used density functional theory calculations to calculate the formation energies of antisite pairs at different separations for four families of pyrochlores. Interestingly, they found titanium-based pyrochlores (titanates) exhibit a different trend from the other three families. For example, the formation energy for faraway antisite pairs increases about $1 \mathrm{eV}$ over that for neighboring pairs in titanates, while in the other three families, the energies essentially exhibit no change. The result suggests that the stability of titanates may depend on how far the antisite pairs are created, which may be tuned by changing irradiation conditions. To support this claim, they used molecular dynamics to conduct cascade simulations to investigate the mean separation of antisites as a function of primary knock-on atom energy (which correlates with irradiation particle energy) and found that the separation did show a dependence on the irradiation conditions. Based on their modeling results, the authors proposed that the stability of titanates can be tuned by adjusting the irradiation spectrum, which could be validated with well-designed target experiments in the future.

The five selected articles in this topic are just a few examples of the recent progress in using advanced characterization and modeling tools to understand the complex radiation effects in oxide ceramics at atomistic and mesoscale levels. Exploring these low-length-scale details can help us understand the underlying mechanisms responsible for the macroscopic degradation behavior in oxide ceramics under radiation. The research activities presented in these articles show that many researchers are working along this direction. They also show that there are many science questions for us to answer in the future. With more dedicated research in this area, we will gain more scientific understanding and use them to design materials to meet the increasing demand on materials performance under radiation. Eventually these efforts will lead to the improvement of nuclear safety and energy conversion efficiency in the future.

The following papers being published under the topic of Radiation Effects in Oxide Ceramics and Novel LWR Fuels provide excellent details and research on the subject. To download any of the papers, follow the url http://link.springer.com/journal/ $11837 / 66 / 12 /$ page/ 1 to the table of contents page for the September 2014 issue (vol. 66, no. 12). • "What Can We Learn from Raman Spectroscopy on Irradiation-Induced Defects in UO2?" L. Desgranges, P. Simon, Ph. Martin, G. Guimbretière, and G. Baldinozzi - "In-situ TEM Observation of Dislocation Evolution in Polycrystalline UO" L.F. He, M. Gupta, M.A. Kirk, J. Pakarinen, J. Gan, and T.R. Allen • "Effect of Grain Boundaries on Krypton Segregation Behavior in Irradiated Uranium Dioxide" Billy Valderrama, Lingfeng He, Hunter B. Henderson, Janne Pakarinen, Brian Jacques, Jian Gan, Darryl P. Butt, Todd R. Allen and Michele V. Manuel • "Using Coupled Mesoscale Experiments and Simulations to Investigate High Burn-up Oxide Fuel Thermal Conductivity Prediction of Irradiation Spectrum Effects in Pyrochlores" Melissa C. Teague, Bradley S. Fromm, Michael R. Tonks, and David P. Field • "Prediction of Irradiation Spectrum Effects in Pyrochlores" B.P. Uberuaga, C. Jiang, C.R. Stanek, K.E. Sickafus, C. Scott, and R. Smith.

\section{REFERENCES}

1. D.J.M. Bevan, I.E. Grey, and B.T.M. Willis, J. Solid State Chem. 61, 1 (1986).

2. D.A. Andersson, J. Lezama, B.P. Uberuaga, C. Deo, and S.D. Conradson, Phys. Rev. B 79, 024110 (2009).

3. L.-F. He, M. Gupta, C.A. Yablinsky, J. Gan, M.A. Kirk, X.M. Bai, J. Pakarinen, and T.R. Allen, J. Nucl. Mater. 443, 71 (2013).

4. S.J. Zinkle, Nucl. Instrum. Meth. B 91, 234 (1994).

5. X.-M. Bai, A.F. Voter, R.G. Hoagland, M. Nastasi, and B.P. Uberuaga, Science 327, 1631 (2010).

6. P.V. Nerikar, D.C. Parfitt, L.A.C. Trujillo, D.A. Andersson, C. Unal, S.B. Sinnott, R.W. Grimes, B.P. Uberuaga, and C.R. Stanek, Phys. Rev. B 84, 174105 (2011).

7. M.R. Tonks, D. Gaston, P.C. Millett, D. Andrs, and P. Talbot, Comput. Mater. Sci. 51, 20 (2012).

8. K.E. Sickafus, L. Minervini, R.W. Grimes, J.A. Valdez, M. Ishimaru, F. Li, K.J. McClellan, and T. Hartmann, Science 289, 748 (2000). 